\title{
Factores de riesgo para cáncer cervicouterino en mujeres de Zacatecas
}

\author{
Maura Sara C astañeda-Iñiguez, M.C ., M. en C., (1) \\ Rebeca Toledo-Cisneros, M.C., (2) Mario Aguilera-D elgadillo, M.C. ${ }^{(3)}$
}

\section{Castañeda-Iñiguez MS, Toledo-Cisneros R, A guilera-Delgadillo $M$. Factores de riesgo para cáncer cervicouterino en mujeres de Zacatecas. Salud Publica Mex 1998;40:330-338.}

\begin{abstract}
Resumen
Objetivo. Conocer las características sociodemográficas reproductivas y de conducta sexual de las usuarias del Programa de Prevención y Control del Cáncer Cervicouterino e identificar los factores de riesgo para dicha patología. Material y métodos. Se hizo un estudio de casos y controles en usuarias del Programa de Prevención y Control del Cáncer Cervicouterino en Zacatecas; los casos fueron todas aquellas pacientes con diagnóstico corroborado por histopatología de displasia severa, cáncer in situ e invasor (251 en total), referidas a la Clínica de Displasias (dependiente del mismo programa), durante el bienio 1993-1995. Los controles se seleccionaron aleatoriamente de entre la población con citología exfoliativa negativa y que era usuaria de dicho programa. Se seleccionó un control por caso, y se le pareó por edad y por la fecha en que se realizó la citología del caso. Resultados. El riesgo de neoplasia cervical aumentó con el número de gestaciones (RM 5.2, IC 95\% 2.6-10.5) para aquellas mujeres con más de 12 gestaciones en relación con las que tenían menos de tres. Por otra parte, el riesgo de neoplasia cervical se incrementa en la medida en que aumentan los partos.Así, las mujeres que tuvieron 120 más partos corren un riesgo cinco veces superior que aquellas que dieron a luz menos de tres veces (RM 5.1, IC 95\% 2.4-11.0). El inicio de las relaciones sexuales en edad temprana está asociado al riesgo de neoplasia cervical; así, las mujeres que postergaron el inicio de sus relaciones sexuales hasta después de los 19 años tuvieron dos veces menos riesgo que quienes comenzaron antes de los 15 años. El uso de anticonceptivos hormonales aumentó el riesgo de cáncer cervical en relación con las mujeres que utilizaron anticonceptivos no hormonales (RM 1.9, IC 95\% 1.3-3.4).
\end{abstract}

\author{
Castañeda-Iñiguez MS, Toledo-Cisneros R, \\ Aguilera-Delgadillo $M$. \\ Risk factors for cervical cancer \\ in women from Zacatecas, Mexico. \\ Salud Publica Mex 1998;40:330-338.
}

\begin{abstract}
A bstract
Objective. To determine the sociodemographic characteristics and the reproductive and sexual behavior of participants in the Cervical $\mathrm{C}$ ancer Prevention and Control Program and to identify the risk factors for this neoplasm. Material and methods. A case control study was performed among participants in the Cervical $\mathrm{C}$ ancer Prevention and Control Program in the state of Zacatecas. The cases consisted of all patients diagnosed and corroborated by histopathology with CIN III and invasive carcinoma of the uterine cervix (251) who were referred to the Dysplasia Clinic (within the same program), during 1993-1995.C ontrols were women randomly selected from the population which participates in this program, who had negative cervical cytology. 0 ne control was selected for each case and paired by age and date, simultaneous to the cervical cytolo gy. Results. Risk of cervical cancer was higher in women with greater number of pregnancies (more than 12 pregnancies) than in women with less than 3 (O R 5.2, CI 95\% 2.6-10.5). This is also true of women with greater number of births (12 or more) who have a risk five times higher than women with less than 3. Beginning sexual activity at an early age was associated to the risk of cervical cancer; women who began their sexual activity after the age of 19 had a risk two times lower than those who started before the age of 15.The use of oral contraceptives increased the risk of cervical cancer in relation to the use of non-hormonal contraceptives (OR 1.9, CI 95\% 1.3-3.4). Conclusions. In the present study, the risk factors for cervical cancer in the population participating in the prevention and control program were higher in women with high parity, who began sexual activity at an early age and/or who consumed oral contraceptives.
\end{abstract}

(1) Coordinadora del Programa de Prevención y Control del Cáncer Cervicouterino y Mamario, Servicios de Salud en Zacatecas, México; investigadora del Centro de Investigación en Salud Poblacional, Instituto N acional de Salud Pública, México.

(2) Jefa del Laboratorio Estatal de Citología, Servicios de Salud en Zacatecas, México.

(3) Coordinador de la Clínica de Displasias, SSA, Servicios de Salud en Zacatecas, México.

Fecha de recibido: 23 de septiembre de 1997 - Fecha de aprobado: 2 de abril de 1998

Solicitud de sobretiros: Dra. Maura Sara Castañeda Iñiguez. Centro de Investigación en Salud Poblacional, Instituto N acional de Salud Pública. Av. Universidad 655, colonia Santa María A huacatitlán, 62508 Cuernavaca, Morelos, México. 
Conclusiones. El estudio de factores de riesgo para neoplasia cervical en la población usuaria del programa de prevención y control mostró que las mujeres con alta paridad, con inicio precoz de relaciones sexuales y las que consumen anticonceptivos de tipo hormonal constituyen una subpoblación con mayor riesgo de padecer neoplasia cervical.

Palabras clave: neoplasmas del cuello uterino; factores de riesgo; estudios de casos y controles; México
Key words:cervix neoplasms; risk factors; case-control studies; Mexico
$L$ os tumores genitales femeninos constituyen aproximadamente una cuarta parte de todos los tipos de cáncer que padecen las mujeres, con excepción del de mama, en el mundo entero. El cáncer cervicouterino representa $15 \%$ de todas las variedades de cáncer diagnosticadas en mujeres y el segundo tipo de cáncer más común en el sexo femenino. Se calcula que su frecuencia es de aproximadamente 465000 nuevos casos cada año, de los cuales 95000 se presentarán en los países desarrollados y 370000 en las naciones en desarrollo (aproximadamente 80\%), donde sigue siendo el tipo de cáncer más importante. ${ }^{1-3}$

Al analizar las tasas de mortalidad por cáncer cervical en diferentes países, se observa que la variación de la mortalidad es notable con tasas que llegan a ser ocho veces más altas en los países de "alto riesgo" en Latinoamérica, el sudeste asiático y África, respecto a algunas naciones de Europa, a Japón y a Australia. ${ }^{4}$ Asimismo, cuando se comparan las tasas de mortalidad ajustadas de Canadá y Estados Unidos de América (EUA), con las de los países de América Latina cuya información sobre mortalidad es confiable (Colombia, Venezuela, Costa Rica), se hace evidente que dichas tasas son más altas en estos últimos pues, además de la elevada cifra de defunciones por cáncer cervicouterino que presentan, ocupan asimismo los primeros lugares en mortalidad por tumores malignos. Inclusive, en las últimas décadas se ha observado un aumento en la mortalidad por cáncer del cérvix en países como Chile, Costa Rica y México.5,6

Las tasas de mortalidad por cáncer en México muestran una marcada tendencia creciente en las últimas décadas. Los tumores malignos en México ocupan el segundo lugar como causa de muerte en la población general y, dentro de éstos, el cáncer cervical representa el tercer lugar como causa de defunción por neoplasias malignas y el primer lugar si se toma en cuenta sólo el sexo femenino, con un total de 4526 defunciones durante 1996, únicamente antecedido por los tumores de pulmón y estómago en primero y segundo lugar, respectivamente. Asimismo, dicha neoplasia constituye $21.4 \%$ del total de las neoplasias malignas, 35\% de las que afectan a la mujer y $85 \%$ de las ginecológicas. ${ }^{7,8}$

A pesar de que las actividades de prevención y control del cáncer cervical se iniciaron en 1975, las tasas de mortalidad por esta neoplasia persisten con tendencias ascendentes: la tasa de mortalidad por cáncer cervical en México, en la presente década, es de 9.5/100 000 mujeres. ${ }^{9}$ Cabe mencionar, además, que en un análisis de la mortalidad por dicha patología realizado en México, ${ }^{10}$ se observó que ese aumento se ha dado principalmente en los grupos de mujeres de mayor edad.

La incidencia de cáncer cervical en México entre 1975 y 1992, con base en las estadísticas de la Secretaría de Salud (SSA), presenta una tendencia ascendente, con una tasa de 9.72 por 100000 mujeres de 15-69 años (bajo cobertura de la SSA) al principio del periodo, y de 42.99 en $1992 .{ }^{11}$ En 1993 se presentaron en el país 6248 casos nuevos de cáncer cervicouterino, cuya mayoría se concentró en los grupos de edad de 35-54 años. ${ }^{12}$

De acuerdo con los resultados obtenidos en diversos estudios epidemiológicos, los factores asociados en forma más consistente con el cáncer cervicouterino son la infección por el virus del papiloma humano (VPH), los hábitos sexuales (antecedentes de dos o más parejas sexuales o el inicio de la vida sexual a edad temprana), los factores de riesgo reproductivo en la mujer (como la multiparidad vaginal o el consumo de anticonceptivos de tipo hormonal) y otros como el hábito tabáquico, que aún es controversial. ${ }^{13-15}$ En este trabajo se presentan los resultados de un estudio de casos y controles realizado con usuarias del Programa de Prevención y Control del Cáncer Cervicouterino en Zacatecas, diseñado para evaluar la relación entre los factores reproductivos y sexuales y el riesgo de neoplasia cervical en dichas pacientes.

\section{Material y métodos}

Se realizó un estudio de casos y controles en el marco del Programa de Prevención y Control del Cáncer 
Cervicouterino de la Secretaría de Salud en el estado de Zacatecas, de enero de 1993 a junio de 1995.

\section{Obtención de casos y controles}

Se identificaron como casos las 251 pacientes diagnosticadas con histopatología con displasia severa, cáncer in situ y carcinoma microinvasor o invasor, referidas a la Clínica de Displasias dependiente de dicho programa durante el periodo citado. El grupo control se seleccionó aleatoriamente por medio de una tabla de números aleatorios entre las pacientes diagnosticadas como negativas (sin imagen citológica de infección viral ni de displasia), usuarias del mismo programa; se pareó cada control con su respectivo caso por edad y año en el que se realizó la citología del caso.

\section{Obtención de la información}

La información se obtuvo de manera retrospectiva, tanto para los casos como para los controles, mediante la hoja de citología ginecológica (que se emplea de manera estandarizada para la recolección de datos de todas las mujeres que acuden a realizarse la prueba del Papanicolaou -PAP-a las unidades médicas de la SSA), de la cual se extrajeron los datos sobre los antecedentes sociodemográficos, sexuales y reproductivos de dichas mujeres. Las variables de estudio se subdividieron en: a) sociodemográficas: edad, procedencia, estado civil y nivel educativo; b) las relacionadas con los factores reproductivos y sexuales: edad de inicio de la vida sexual activa, número de compañeros sexuales, embarazos, partos, cesáreas y abortos, además de la presencia de signos citológicos positivos de infección por VPH, y c) otras como el uso de anticonceptivos.

\section{A nálisis estadístico}

A partir de los 251 casos estudiados con neoplasia cervical confirmada histológicamente y los 251 controles, se realizaron análisis univariados, bivariados y multivariados mediante el paquete estadístico SPSS versión 6.0. ${ }^{16}$ Para estimar el riesgo de neoplasia cervical asociado a los diferentes factores de riesgo se calculó la razón de productos cruzados o razón de momios (RM) como aproximación del riesgo relativo e intervalos de confianza (IC) al 95\%. ${ }^{17}$ Para controlar los efectos de los potenciales factores confusores se utilizó la estratificación y el procedimiento de Mantel-Haenszel, además de la regresión logística condicional (en el modelo final se incluyeron las variables anticoncepción, número de partos vaginales y edad de inicio de la vida sexual, mismas que se seleccionaron por su plausibilidad biológica y/o por ser estadísticamente significativas). ${ }^{18}$

\section{Resultados}

En relación con la patología presentada y corroborada por histopatología, los casos se distribuyeron de la siguiente manera: $59 \%$ padecía displasia severa; $37 \%$, cáncer in situ, y 4\%, cáncer microinvasor e invasor. Respecto a las características sociodemográficas de las participantes, no se observaron diferencias de edad entre casos (media $=40.5)$ y controles (media $=40.6)$, lo cual era esperado debido al proceso de pareamiento por edad utilizado en la obtención de los controles. Respecto a las variables procedencia, estado civil y nivel educativo no se observan diferencias significativas entre casos y controles.

En lo concerniente a las variables relacionadas tanto con la reproducción como con el comportamiento sexual de ambos grupos, se observó similitud en las edades de la menarca de los casos (media $=13.3$ ) y los controles (media=13.1) (cuadro I). La media de edad de inicio de la vida sexual activa entre los casos fue un año más temprana que en los controles (17.8 y 18.9, respectivamente), con un valor de $p<0.05$. La media en el número de compañeros sexuales fue más alta en los casos (1.2) que en los controles (1.0). La media en el número de embarazos y partos para los casos $(7.4 \mathrm{y}$ 6.1, respectivamente) fue mayor que para los controles (6.4 y 5.2, respectivamente), con una $p<0.05$. Hubo menos cesáreas en los casos que en los controles (media $=0.14$ y 0.18 , respectivamente) y ambos presentaron menos de una cesárea por mujer como promedio. En relación con el número de abortos, la media tanto para casos como para controles fue inferior a un aborto por mujer (0.81 y 0.74 , respectivamente).

En el cuadro II se presentan los resultados del análisis bivariado de los factores de riesgo ya mencionados. El riesgo de neoplasia cervical es mayor en la medida en que el inicio de la vida sexual activa es más temprano; así, al tomar como referencia a las mujeres que iniciaron su vida sexual después de los 20 años, se estima que aquellas que lo hicieron antes de los 15 años tienen un riesgo dos veces mayor (RM 2.0, IC95\% 1.2-3.5). Respecto al número de compañeros sexuales no se observan diferencias significativas entre aquellas que indicaron haber tenido una pareja sexual y aquellas que notificaron más de una (RM 0.8, IC95\% 0.4-0.6). Por otra parte, el aumento en el número de gestaciones ha mostrado una tendencia lineal en el riesgo de neoplasia cervical (test de tendencia con un valor de $p<0.0001$ ). Así, las mujeres que han tenido más de doce gestaciones corren un riesgo cinco veces 
Factores de RIESGo asociados

A NEOPLASIA CERVICAL.

ZACATECAS, 1993-1995

Mínima Máxima Media DE $p^{*}$

Edad de la menarca

\begin{tabular}{llllll} 
Casos & 13 & 18 & 13.3 & 1.3 & 0.2703 \\
\hline Controles & 10 & 18 & 13.1 & 1.8 &
\end{tabular}

Edad de inicio de vida sexual

\begin{tabular}{llllll} 
Casos & 13 & 29 & 17.8 & 2.8 & 0.0011 \\
\hline Controles & 13 & 43 & 18.9 & 4.4 &
\end{tabular}

N úmero de parejas sexuales

\begin{tabular}{lrrrrr} 
Casos & 1 & 10 & 1.2 & 1.2 & 0.0496 \\
\hline Controles & 1 & 3 & 1.0 & .37 &
\end{tabular}

N úmero de embarazos

\begin{tabular}{llllll} 
Casos & 0 & 20 & 7.4 & 4.2 & 0.0004 \\
\hline Controles & 0 & 20 & 6.1 & 4.1 &
\end{tabular}

N úmero de partos vaginales

\begin{tabular}{llllll} 
Casos & 0 & 19 & 6.4 & 3.8 & 0.0003 \\
\hline Controles & 0 & 19 & 5.2 & 3.7 &
\end{tabular}

Número de abortos

\begin{tabular}{lrrrrr} 
Casos & 0 & 7 & .81 & 1.2 & 0.4780 \\
\hline Controles & 0 & 10 & .74 & 1.3 &
\end{tabular}

N úmero de cesáreas

\begin{tabular}{llllll} 
Casos & 0 & 3 & .14 & .41 & 0.3307 \\
\hline Controles & 0 & 3 & .18 & .50
\end{tabular}

* p de prueba t para diferencia de medias

superior de contraer este tumor maligno que aquellas que se han embarazado menos de tres veces (RM 5.2, IC95\% 2.6-10.5). Esta situación se repite respecto al número de partos, pues el riesgo se quintuplica para las mujeres que han tenido 12 o más en relación con aquellas que sólo tuvieron menos de tres (RM 5.1, IC95\% 2.4-11.0); la tendencia es igualmente lineal (test de tendencia con un valor de $p<0.0001$ ). Respecto al número de cesáreas y abortos en quienes participaron en el estudio, no se observan diferencias significativas entre aquellas que notificaron una cesárea y/o aborto y aquellas que indicaron más de uno (cuadro II).

El uso de métodos de planificación familiar como es el consumo de anticonceptivos de tipo hormonal (RM 1.65, IC95\% 0.9-3.0), el uso del dispositivo intrau-

\section{Cuadro II \\ Modelo bivariado de FACTORES DE RIESGO \\ ASOCIADOS A NEOPLASIA CERVICAL. ZACATECAS, 1993-1995}

Casos Controles $\quad \mathrm{RM}^{\ddagger} \quad \mathrm{IC} 95 \%$

Edad de inicio vida sexual activa

\begin{tabular}{lllll}
$>20^{*}$ & 60 & 89 & 1.0 & \\
\hline $18-19$ & 64 & 55 & 1.7 & $1.1-2.8$ \\
\hline 17 & 37 & 38 & 1.4 & $0.8-2.5$ \\
\hline 16 & 38 & 31 & 1.8 & $1.0-3.3$ \\
\hline$<15$ & 52 & 38 & 2.0 & $1.2-3.5$
\end{tabular}

$N$ úmero de compañeros sexuales

\begin{tabular}{lrrrr}
$0-1^{*}$ & 231 & 228 & 1.0 & \\
\hline$>1$ & 20 & 23 & 0.8 & $0.4-1.6$
\end{tabular}

N úmero de gestaciones

\begin{tabular}{lrrrr}
$0-3^{*}$ & 46 & 83 & 1.0 & \\
\hline $4-7$ & 102 & 92 & 2.4 & $1.5-3.9$ \\
\hline $8-11$ & 51 & 46 & 3.2 & $1.7-6.1$ \\
\hline$>12$ & 52 & 30 & 5.2 & $2.6-10.5$
\end{tabular}

Valor $p$ tendencia 0.0001

N úmero de partos

\begin{tabular}{crrrr}
$0-3^{*}$ & 60 & 99 & 1.0 & \\
\hline $4-7$ & 102 & 89 & 2.4 & $1.5-4.0$ \\
\hline $8-11$ & 58 & 46 & 3.4 & $1.9-6.4$ \\
\hline$>12$ & 31 & 17 & 5.1 & $2.4-11.0$
\end{tabular}

Valor $p$ tendencia 0.0002

N úmero de cesáreas

\begin{tabular}{rrrrr}
$<1^{*}$ & 221 & 217 & 1.0 & \\
\hline$>1$ & 30 & 34 & 1.1 & $0.6-2.0$
\end{tabular}

N úmero de abortos

\begin{tabular}{rrrrr}
$<1^{*}$ & 144 & 154 & 1.0 & \\
\hline$>1$ & 107 & 97 & 1.1 & $0.8-1.7$
\end{tabular}

* Grupo de referencia

₹ RM ajustada por edad

$\S$ Test de tendencia lineal

terino (RM 1.15, IC95\% 0.7-2.0) y la salpingoclasia (RM 1.11, IC95\% 0.6-1.9) no se asociaron al riesgo de neoplasia cervical, como se puede observar en el cuadro III. La prevalencia de uso de anticonceptivos de barrera o locales fue mayor en las mujeres seleccionadas como controles; asimismo, se observó un efecto protector de riesgo de enfermedad (RM 0.14, IC95\% 0.0-0.4) entre quienes usaban dicho anticonceptivo. 
Al ajustar por edad, edad de inicio de la vida sexual, número de partos vaginales y número de parejas sexuales (cuadro IV), se puede observar que el consumo de anticonceptivos de tipo hormonal aumenta el riesgo de neoplasia cervical respecto a las mujeres que re-

\section{Cuadro III \\ Anticonceptivo utilizado ASOCIADO A RIESGO \\ DE NEOPLASIA CERVICAL. ZACATECAS, 1993-1995}

$\%$ casos $\quad \%$ controles $\quad \mathrm{RM}^{\ddagger} \quad \mathrm{IC} 95 \%$

Tipo

\begin{tabular}{lrrrr} 
N inguno* & 40.6 & 36.5 & 1.0 & \\
\hline Hormonales & 17.9 & 12.7 & 1.65 & $0.9-3.0$ \\
\hline DIU & 19.6 & 20.6 & 1.15 & $0.7-2.0$ \\
\hline OTB & 17.5 & 16.5 & 1.11 & $0.6-1.9$ \\
\hline Locales & 4.4 & 13.6 & 0.14 & $0.0-0.4$
\end{tabular}

* Grupo de referencia

₹ RM ajustada por edad, edad de inicio de vida sexual, número de partos vaginales, número de parejas sexuales

OTB: oclusión tubaria bilatera curren a los anticonceptivos que no son de origen hormonal (RM 1.9, IC95\% 1.1-3.4). Como ya se mencionó, el riesgo de que aparezca una neoplasia cervical se incrementa conforme aumenta el número de partos vaginales. Ese riesgo aumenta también en relación con la edad de inicio de la vida sexual activa; así, tomando como referencia a las mujeres que comenzaron a tener relaciones sexuales después de los 20 años, se estima que aquellas que comenzaron antes de los 15 años corren un riesgo dos veces mayor (RM 2.1, IC95\% 1.3-3.6).

$\mathrm{Al}$ ajustar por otros posibles factores confusores como son procedencia y nivel educativo, además de los ya mencionados, no se observan modificaciones significativas en el riesgo de contraer neoplasia cervical en lo que respecta a la variable anticoncepción y edad de inicio de la vida sexual. Sin embargo, en el caso del número de partos la magnitud de la estimación sí se modifica al ajustarla por dichas variables.

En el cuadro V se muestra que la presencia de signos positivos de VPH por diagnóstico citológico se asoció significativamente con el riesgo de padecer neoplasia cervical, de tal manera que las mujeres que las presentaron corren un riesgo cinco veces superior que aquellas que no las tuvieron (RM 5.2, IC95\% 2.0-9.4).

\section{Cuadro IV \\ Modelo MUltivariado de factores de RIESGo ASOCIADOS A NeOPLASIA CERVICAL. ZACATECAS, 1993-1995}

\begin{tabular}{|c|c|c|c|c|c|c|c|c|}
\hline & Casos & $\%$ & Controles & $\%$ & $R M_{(a)}$ & IC95\% & $\mathrm{RM}_{(A)}$ & IC95\% \\
\hline \multicolumn{9}{|l|}{ Anticoncepción* } \\
\hline No hormonal & 97 & 68.3 & 123 & 79.8 & 1.0 & & 1.0 & \\
\hline Hormonal & 45 & 31.7 & 31 & 20.2 & 1.9 & $1.1-3.4$ & 1.9 & $1.1-3.4$ \\
\hline
\end{tabular}

N úmero de partos vaginales

\begin{tabular}{lrrrrrrrrr}
$0-3$ & 60 & 23.4 & 99 & 39.4 & 1.0 & & 1.0 & 1.9 & $1.2-3.1$ \\
\hline $4-7$ & 102 & 40.2 & 89 & 35.4 & 2.4 & $1.5-3.9$ & 1.9 \\
\hline $8-11$ & 58 & 23.1 & 46 & 18.4 & 3.2 & $1.7-6.1$ & 2.0 & $1.1-3.5$ \\
\hline$\geq 12$ & 31 & 12.3 & 17 & 6.8 & 5.2 & $2.6-10.5$ & 3.8 & $2.1-7.0$
\end{tabular}

Edad de inicio de vida sexuals

\begin{tabular}{lllllllll}
$\geq 20$ & 60 & 23.9 & 89 & 35.4 & 1.0 & & 1.0 & 1.7 \\
\hline $18-19$ & 64 & 25.5 & 55 & 21.9 & 1.7 & $1.1-2.9$ & 1.7 & 1.3 .0 \\
\hline 17 & 37 & 14.7 & 38 & 15.2 & 1.4 & $0.8-2.5$ & 1.4 & $0.8-2.6$ \\
\hline 16 & 38 & 15.2 & 31 & 12.3 & 1.8 & $1.1-3.4$ & 1.7 & $1.0-3.0$ \\
\hline$\leq 15$ & 52 & 20.7 & 38 & 15.2 & 2.1 & $1.3-3.6$ & 2.0 & $1.2-3.5$
\end{tabular}

\footnotetext{
$\mathrm{RM}_{(\underset{*}{*})}$

* RM ajustada por edad, edad de inicio de vida sexual, número de partos, número de compañeros sexuales

₹ RM ajustada por edad, edad de inicio de vida sexual, número de compañeros sexuales, anticoncepción

§ RM ajustada por edad, número de compañeros sexuales y anticoncepción

$\mathrm{RM}_{(\mathrm{A})} \mathrm{RM}$ ajustada por las anteriores además de procedencia y nivel educativo
} 


\section{Cuadro V \\ Presencia de signos citológicos positivos de INFECCIÓN POR VPH ASOCIADO A RIESGO DE NEOPLASIA CERVICAL. ZACATECAS, 1993-1995}

\begin{tabular}{|c|c|c|c|c|c|c|}
\hline Signos de VPH & Casos & $\%$ & Controles & $\%$ & $\mathrm{RM} *$ & IC95\% \\
\hline N egativos & 179 & 71.4 & 233 & 92.8 & 1.0 & \\
\hline Positivos & 72 & 28.6 & 18 & 7.2 & 5.2 & $2.9-9.4$ \\
\hline
\end{tabular}

\section{Discusión}

En este estudio se utilizó una metodología de casos y controles para investigar los factores de riesgo que influyen en el desarrollo de la neoplasia cervical, en mujeres usuarias del Programa de Prevención y Control del Cáncer Cervicouterino de la SSA en el estado de Zacatecas. Se eligió dicha metodología en razón de su factibilidad, aunque contemplando asimismo sus posibles limitaciones ya que, por ejemplo, el uso de un instrumento de recolección de datos como la hoja de citología ginecológica dificulta la interpretación correcta de la información allí proporcionada.

Al comparar la media de edad en mujeres con neoplasia cervical en este estudio, con las que informan otros autores, se observa que en México, al igual que en los países en desarrollo, dicha patología es más frecuente entre la tercera y cuarta década de la vida, ${ }^{19-22}$ en comparación con lo que sucede en los países desarrollados, donde se presenta con mayor frecuencia a partir de los 50 años. ${ }^{23,24}$ Dadas las características geográficas y de servicios de salud prevalecientes en el estado de Zacatecas, el porcentaje de participantes en el estudio de procedencia rural es más alto que en el de la urbana, hecho seguramente determinado por el tipo de población que atiende la SSA, es decir, por la llamada "población abierta". Aunque algunos estudios en Latinoamérica notifican que el riesgo relativo de las mujeres a padecer cáncer cervical aumentó en relación inversa al número de años de educación, ${ }^{25}$ en el presente estudio y en otros realizados en diversas áreas, dicha relación no fue estadísticamente significativa. ${ }^{26}$

En este estudio y en coincidencia con lo que notifica al respecto la SSA, ${ }^{27}$ las mujeres con más de siete embarazos representan más de $40 \%$, hecho que posiblemente influye en los hallazgos, pues se observa una tendencia lineal de riesgo a partir del primer embarazo, de modo tal que el riesgo de las mujeres con $12 \mathrm{o}$ más embarazos fue cinco veces mayor. Una probable explicación se fundamenta en el hecho de que el embarazo provoca un estado de inmunosupresión que podría aumentar la susceptibilidad del organismo a los agentes infecciosos. Se ha señalado que la neoplasia cervical aparece con mayor frecuencia en el labio anterior del cérvix, zona donde el traumatismo obstétrico es más intenso, por lo que es plausible considerar al parto vaginal como un factor de riesgo de cáncer cervicouterino. ${ }^{28}$ Una vez que se ajustó por diversos factores de confusión, en este trabajo se encontró que la $\mathrm{RM}$ de las mujeres con 12 o más partos vaginales se incrementa notablemente en relación con aquellas que han tenido menos de tres. Aunque en varios estudios previos se ha descartado la paridad como factor de riesgo de cáncer cervical, en diversas investigaciones se ha demostrado que el riesgo aumenta con la paridad, después de ajustar por diversos factores. ${ }^{20}$ Lo anterior se explica en función de la incapacidad de algunos estudios previos para detectar aumento en el riesgo debido, tal vez, a que entre las mujeres había pocas con un antecedente de múltiples embarazos. Ese no es el caso de los estudios realizados en países latinoamericanos $^{29,30}$ y en mujeres latinas residentes en EUA, ${ }^{31}$ donde se ha obtenido una estimación de riesgo de cáncer cervical similar, con la presencia de seis o más partos vaginales, por lo que se puede considerar la multiparidad vaginal como un factor de riesgo importante para contraer el padecimiento. Cabe mencionar que en otros estudios ${ }^{32}$ el efecto de la paridad fue moderado y/o no consistente.

El inicio temprano de las relaciones sexuales se ha asociado, en numerosos estudios, con el incremento en el riesgo de padecer cáncer cervical: esa relación se ha explicado con base en la consideración de que la zona de transformación del epitelio cervical, la más proliferativa durante la pubertad y la adolescencia (el llamado "periodo vulnerable"), es especialmente susceptible a alteraciones que pueden ser inducidas por agentes transmitidos sexualmente, entre ellos el VPH. Lo anterior es congruente con la idea de que las infecciones por VPH durante la adolescencia tienen una probabilidad más alta de convertirse en infecciones crónicas y que implican un mayor riesgo de contraer cáncer cervical. Ese riesgo se reduce al postergar el inicio de las relaciones sexuales. ${ }^{33,34}$

En este estudio se observó que el incremento en el riesgo de que se presente la neoplasia cervical cuando la vida sexual da comienzo antes de los 15 años de edad se duplica respecto a la posibilidad de haber postergado esa actividad hasta después de los 19 años, y que es importante señalar que esta asociación es independiente del número de compañeros sexuales, ya que no 
varió al ajustarla por esa variable. Existen diversos estudios realizados en México que coinciden con estos resultados. ${ }^{35}$

En la mayoría de los trabajos mencionados la asociación entre un elevado número de compañeros sexuales y la aparición de cáncer cervical está presente, y se considera que dicho número es una medida sustitutiva de la exposición a infección por VPH. Sin embargo, en este estudio esa asociación no fue estadísticamente significativa, debido quizás a que la información que dieron las participantes sobre el número de compañeros sexuales que han tenido es poco confiable, puesto que los datos fueron recolectados por distintas personas, en un marco tal vez no apropiado para responder de manera fiable a dicha pregunta, y/ o a que el número de compañeros sexuales generalmente es un tema tabú en México (donde la monogamia entre las mujeres suele ser la regla), hecho que de alguna manera imposibilita evitar el sesgo de información al responder a dicha pregunta.

En este estudio el porcentaje de mujeres que utilizan algún método anticonceptivo es parecido al que indican otros estudios realizados en México, ${ }^{36}$ aunque el porcentaje de utilización varía por tipo de anticonceptivo; en este caso, y debido a las políticas sanitarias de las autoridades de salud mexicanas, el dispositivo intrauterino fue el método más utilizado y, si bien el consumo de anticonceptivos de tipo hormonal fue más importante en los casos que en los controles, el incremento en el riesgo de neoplasia cervical entre quienes utilizaron dichos anticonceptivos estuvo en el límite de la significación estadística. Al ajustar por probables factores de confusión como el inicio temprano de relaciones sexuales o el número de partos, procedencia y escolaridad, la asociación no varió.

Cabe considerar las limitaciones que respecto a la variable tipo de anticonceptivo utilizado tiene este estudio, ya que fue imposible investigar la duración en tiempo del uso de anticonceptivos, sobre todo los de tipo hormonal, así como el tipo y la categoría de uso (nunca, alguna vez), por lo que es difícil establecer una posible asociación entre el uso de los de tipo hormonal y una neoplasia cervical. Los resultados que se presentan ahora coinciden con los de otros estudios, donde no se observó un incremento importante en el riesgo de padecer cáncer cervical en las mujeres que utilizaban anticonceptivos de tipo hormonal, ${ }^{37} \mathrm{o}$ bien, éste fue insignificante. ${ }^{38}$ No obstante, en otros estudios ${ }^{25}$ se sugiere la existencia de una interacción entre el uso de anticonceptivos de tipo hormonal y la infección por VPH en el desarrollo de cáncer cervical (el descubrimiento de ese efecto en las mujeres positivas a VPH mas no en las que tuvieron resultados negativos parece indicar que el efecto más probable de este tipo de anticonceptivos es el de promover la transición de la infección crónica por VPH a la neoplasia cervical).

Se considera que la citología es una técnica poco sensible para detectar la presencia de $\mathrm{VPH}$, ya que sólo puede identificar correctamente $15 \%$ del total de casos ADN VPH positivos, ${ }^{39}$ empero, diversos estudios que se han hecho en poblaciones que acuden a los programas de detección o a clínicas de atención de enfermedades de transmisión sexual, notifican prevalencias que van desde $0.24 \%$ hasta $9-13 \%$, en función del tipo de población estudiada. ${ }^{40}$ En este caso el porcentaje de presencia de signos positivos de $\mathrm{VPH}$ por citología es más alto en los casos que en los controles (28.6 y $7.1 \%$, respectivamente). Sin embargo, el porcentaje de casos con la presencia de signos de VPH en la citología podría considerarse bajo, sobre todo tomando en cuenta que se trata de la población abierta y no de la considerada de alto riesgo para la adquisición de enfermedades de transmisión sexual. Tamayo $^{36}$ notifica en un estudio de infección por VPH en mujeres con y sin citología anormal, que $4.6 \%$ de las 3257 mujeres estudiadas presentaban evidencia citológica de infección cervical por VPH, cifras que son difíciles de comparar ya que se trata de poblaciones con características diferentes. Es necesario destacar que desafortunadamente fue imposible corroborar la presencia de VPH mediante el análisis histológico.

Dentro de las limitaciones de este trabajo vale la pena mencionar la imposibilidad de generalizar sus resultados al resto de la población, puesto que se desarrolló dentro del marco de un programa de prevención y control de cáncer cervicouterino, de tal manera que la población estudiada no necesariamente es representativa de las mujeres del estado de Zacatecas. Además, la recolección de datos mediante la hoja de citología pudo dar lugar a sesgos de información y de memoria, pues la tarea la llevó a cabo personal de salud muy diverso (médicos, enfermeras, auxiliares de salud), lo cual dificultó la obtención de la información sobre todo en lo relacionado con la actividad sexual de las participantes. Asimismo, la selección del grupo control fue de alguna manera artificial, ya que se consideró la ausencia de alteraciones en una sola laminilla como sinónimo de normalidad. Fue imposible obtener los antecedentes del PAP, ya que el instrumento utilizado para la recolección de la información no los especifica; sin embargo, ese dato habría sido muy interesante, ya que está bien documentado que aquellas mujeres que no se han realizado ningún PAP con anterioridad corren mayor riesgo de tener cáncer cervical. El hecho de sólo contar con un diag- 
nóstico citológico de la presencia de signos de infección por VPH dificulta el análisis ya que, si bien su porcentaje es más alto entre los casos que en los controles, resulta difícil establecer claramente una asociación con la presencia de neoplasia cervical dadas las limitaciones de dicha técnica para detectar realmente la presencia de VPH. Queda claro que es necesario, en futuros estudios, utilizar técnicas como la reacción en cadena de la polimerasa, que es el método más sensible para la identificación de VPH, así como de sus diferentes subtipos.

El estudio de factores de riesgo para cáncer cervicouterino en una muestra de mujeres del estado de Zacatecas hizo evidente que las que han tenido un número elevado de embarazos, alta paridad, un inicio precoz de relaciones sexuales y las consumidoras de anticonceptivos de tipo hormonal constituyen una subpoblación con mayor riesgo de presentar neoplasia cervical. Asimismo, la información que se obtuvo para realizar este estudio es relevante, ya que proporciona una base para la identificación de mujeres de alto riesgo, misma que debe utilizarse para replantear el Programa de Prevención y Control del Cáncer Cervicouterino en los estados de la República.

\section{Agradecimientos}

Se agradece a la doctora Silvia de Sanjosé el gran apoyo y la valiosa colaboración que brindó para la realización de este trabajo. Asimismo se reconoce el valioso apoyo que aportó el resto del equipo de trabajo del Servei d’Epidemiologia y Registre de Cancer del Institut Oncòlogic Duran y Reynals de la ciudad de Barcelona y el Instituto de Cooperación Iberoamericana.

\section{Referencias}

1. Borrás JM, Iglesias X. Cribado del cáncer de cuello del útero. Med Clin1994:1:102-108.

2. Parkin DM, Pisani P, Ferlay J. Estimates of the worldwide incidence of eighteen major cancers in 1985. Int J C ancer 1993;54:594-606.

3. Vizcaino AP, Moreno V, Bosch FX, Muñoz N, Barros-Dios X, Parkin D. International trends in the incidence of cervical cancer: 1. Adenocarcinoma and adenosquamous cell carcinomas. Int J Cancer 1998;75(4):536-545. 4. Boyle P. G lobal burden of cancer. Lancet 1997;349 suppl II:23-26.

5. Restrepo HE. Cancer epidemiology and control in women in Latin A merica and the $\mathrm{C}$ aribbean. En:Pan A merican Health 0 rganization.W omen in health and development. W ashington, DC: PAHO, Publicación Cientifíca, núm. 541, 1993.

6. Robles $C, W$ hite $F$, Peruga A.Tendencias de la mortalidad por cáncer de cuello de útero en las Américas. Bol 0 ficina Sanit Panam 1996;121(6): 478-490.
7. Secretaría de Salud. Principales resultados de la estadística sobre mortalidad en México, 1996. Salud Publica Mex 1997;39(5):486-490.

8. Secretaría de Salud. Cáncer: un encuentro con el futuro epidemiológico. Los retos de la transición. México D.F.: SSA, Cuadernos de Salud, 1994:36-42

9. Secretaría de Salud. Programa Nacional de Prevención y Control del Cáncer Cervicouterino en México. México, D.F.: SSA, 1994:4-5.

10. Lazcano P, Rascón P, Lozano A, Velazco M. Mortality from cervical carcinoma in Mexico: Impact of screening, 1980-1990. Acta Cytol 1996; 40:506-512.

11. Secretaría de Salud. Tumores. México, D.F.: SSA, Series Monográficas, Perfiles Estadísticos, núm. 7, 1993.

12. Secretaría de Salud. Compendio de información sobre morbilidad por cáncer cervicouterino 1972-1992. México, D.F.:D irección General de Epidemiología, 1993.

13. Hakama M.W ho should be screened for cervical cancer? En: Screening of Cervical $C$ ancer For W hom,W hy and How? Experts' Conference. Francia: Monsonego, 1994:2-8.

14. Schiffman M. N ew epidemiology of human papillomavirus infection and cervical neoplasia. J N atl Cancer Inst 1995;87(18):136-139.

15. Lazcano P, Hernández A, López $C$, Alonso-De Ruiz P, Torres L, $G$ onzález $L$ et al. Factores de riesgo reproductivo e historia de vida sexual asociados a cáncer cervical en México. Rev Invest Clin 1995;47(5): 377-385.

16. N orusis M. SPSS for windows: Advanced statistics, Release 6.0. Chicago, 1993.

17. Kahn H, Sempos C. Statistical methods in epidemiology. N ueva York: O xford University Press, 1989:56.

18. Schlesselman J. Case-control studies. D esign, conduct, analysis. N ueva York: O xford University Press, 1982.

19. Mukherjee BM, Sengupta S. A case-control study of reproductive risk factors associated with cervical cancer. Int J C ancer 1994;59(4):476-482. 20. Herrero R, Brinton L, ReevesW, Brenes M, Tenorio F. Sexual behavior, venereal diseases, hygiene practices, and invasive cervical cancer in a highrisk population. Cancer 1990;65:380-386.

21. Herrero R, Brinton L, Reeves W, Brenes M, Tenorio F. Factores de riesgo de carcinoma invasor del cuello uterino en América Latina. Bol 0 ficina Sanit Panam 1990;109(1):7-25.

22. Hernández AM, Lazcano PE, Berumen CJ, Cruz VA, Alonso de RP, González LG. Human papilloma virus 16-18 infection and cervical cancer in Mexico:A case control study. Arch Med Res 1997;28(2):265-271.

23. Parazzini F, La Vecchia C, N egri E. Reproductive factors and the risk of invasive and intraepithelial cervical neoplasia. $\mathrm{Br}$ J Cancer 1989;59: 805-809.

24. Biswas L, Manna B, Maiti P, Sengupta S. Sexual risk factors for cervical cancer among rural Indian women:A case control study. Int J Epidemiol 1997;26(3):491-495.

25. Muñoz N, Bosch FX, De Sanjosé S,Viladiu P,Tormo J, Moreo P et al. El virus del papiloma humano en la etiología del cáncer cervicouterino. PAHO Bull 1993;115:301-309.

26. $O$ Isen $A$, G joen $K$, Sauer $T$, $O$ rstavik, $Y, N$ aess $O$, Magnus $P$. Human papillomavirus and cervical intraepithelial noeplasia grade II-III: A population-based case-control study. Int J C ancer 1995;61:312-315.

27. Secretaría de Salud. Cáncer. Retos de la transición. Un encuentro con el futuro epidemiológico. México, DF:SSA, 1994:27-28.

28. Becker MT, W heeler C, McG ough N, Stidley C, Parmenter C, D orin $M$ et al.C ontraceptive and reproductive risk for cervical dysplasia in Southwestern Hispanic and Non-Hispanic white women. Int J Epidemiol 1994;23(5):913-921.

29. Herrero R, Brinton L, Reeves W, Brenes M, Tenorio F. Factores de riesgo de carcinoma invasor del cuello uterino en América Latina. Bol 0 ficina Sanit Panam 1990;109(1):7-25. 
30. Eluf-N eto J, Booth M, Muñoz N, Bosch FX, Meijer C, W alboomers J. Human papillomavirus and invasive cervical cancer in Brazil. $\mathrm{Br} J$ Cancer 1994;69:114-119.

31. Peters R, Thomas D, H agan D. Risk factors for invasive cervical cancer among Latinas and no Latinas in Los Angeles County. JN CI 1986;77:9411077.

32. Bosch FX, Muñoz N, De Sanjosé S, Izarzugaza I, Gili M,Viladiu P. Risk factors for cervical cancer in Colombia and Spain. Int J Cancer 1992;52: 750-758.

33. Muñoz N, Bosch FX, De Sanjosé S, Shah KV.The role of HPV in the etiology of cervical cancer. Mutat Res 1994;305:293-301.

34. Biswas LM, Manna B, Maiti PK, Sengupta S. Sexual risk factors for cervical cancer among rural Indian women: A case control study. Int J Epidemiol 1997;26(3):491-495.

35. Lazcano P, Rojas R, López A, López C, Hernández M. Factores de riesgo reproductivo y cáncer cérvico-uterino en la $\mathrm{C}$. de México. Salud Publica Mex 1993;35:65-73.
36. Tamayo L, Echaniz A, Cruz V. Infección por el virus del papiloma humano en mujeres con y sin citología cervical anormal. Ginecol 0 bstet Mex 1993:61:27-33.

37. Slattery ML, $O$ verall JC, A bbott TM, French TK, Robison LM, G ardner J. Sexual activity, contraception, genital infections, and cervical cancer: Support for a sexually transmitted disease hypothesis. Am J Epidemiol 1989;130(2):248-257.

38. Schlesselman JJ. Net effect of oral contraceptive use on the risk of cancer in woman in the United States. O bstet Gynecol 1995;85(5): 793-801.

39. Schneider A, Sawada E, Gissmann L, Shah K. Human papillomaviruses in women with a history of abnormal Papanicolaou smear and their male partners. 0 bstet Gynecol 1987;69:554-562.

40. Kjaer S, De Villiers E, Hangaard B, Chistensen R, Teisen C. Human papillomavirus, herpes simplex virus and cervical cancer incidence in Greenland and Denmark. A population-based cross sectional study. Int J Cancer 1989;41:518-524. 